\title{
Quantum remote sensing: a challenge in radiolocation
}

\section{Tomasz Borowski, Mateusz Pasternak, Jerzy Pietrasinski}

Tomasz Borowski, Mateusz Pasternak, Jerzy Pietrasinski, "Quantum remote sensing: a challenge in radiolocation," Proc. SPIE 11442, Radioelectronic Systems Conference 2019, 1144212 (11 February 2020); doi: $10.1117 / 12.2565720$

SPIE. Event: Radioelectronic Systems Conference 2019, 2019, Jachranka, Poland 


\title{
Quantum remote sensing - a challenge in radiolocation
}

\author{
Tomasz Borowski \\ tomasz.borowski@wat.edu.pl \\ Mateusz Pasternak \\ jerzy.pietrasiński\}@,wat.edu.pl \\ Jerzy Pietrasiński \\ jerzy.pietrasiński\}@,wat.edu.pl
}

Military University of Technology, gen. Sylwester Kaliski Str., 200-908 Warszawa, Poland

\begin{abstract}
Conducting research on the state of superposition and the phenomenon of tunnelling has resulted in the discovery of quantum entanglement and quantum teleportation. They have laid the foundation for quantum cryptography and the construction of devices for remote sensing surveillance. The article presents selected aspects and references to some phenomena formulating the basis of quantum remote sensing. The potential benefits of quantum entanglement and quantum entangled photon beam illumination in this field have also been mentioned.
\end{abstract}

Keywords: quantum radar, quantum entanglement, entangled photons, ghost imaging,

\section{INTRODUCTION}

There are many physical phenomena explaining the operation of optical, magnetic, electronic, or nuclear devices. The performance of electronic components such as diodes, transistors, hallotrons, light emitting diodes and lasers at the lowest level is directly attributable to quantum effects. Except for superfluidity, the direct visual observation of quantum effects is not possible. Some of them, such as e.g. superconductivity, tunnelling, or laser scanning, are dynamically integrated with remote sensing techniques (e.g. the phenomenon of Josephson, Hall). Recently, many research centres have been particularly interested in the studies and potential applications of the phenomenon called quantum entanglement. All signs point to the fact that the quantum entanglement method applied in remote sensing will be an important breakthrough in this field of technology. Every radar with a classic design has its own limitations and drawbacks. In addition to the usual noise and nature-related restrictions, radio signals can be disturbed or tracked and eventually destroyed. A system working with quantum entangled particles will avoid all of these limitations. Remote sensing techniques established on a concept exploring the results of detection of extremely strong spectral-time correlations - e.g. wavelength, polarization or the moment and time of object observation - which occur between separate objects, are called quantum remote sensing [1]. An example of such a correlation is the state which is formed as a result of quantum entanglement of photons. The state of entanglement of photons shall be recognised by the degree of random correlation of the results of measurements of those parameters of photons on which the measurement of their correlation has been made, e.g. polarisation. The properties of quantum entangled photons lay foundation for the thesis stating that quantum remote sensing will not only face no obstacles in detecting any objects, but also the thesis that monitoring with the use of a beam of quantum entangled photons will be undetectable and completely resistant to the action of electronic means of combat.

Despite the fact that only until recently quantum remote sensing seemed to be in the sphere of theoretical possibilities rather than a usable solution via ready-made devices, since 2000 the experiments performed as well as publications on quantum entanglement research and patents already granted (mainly in the USA, Japan and China) have been signalling the success of quantum remote sensing and the possibility of its practical use. Please note that the term "quantum radar" is quite commonly used here, although it must be borne in mind that the acronym RADAR is inseparably associated with radio waves, which are not used in quantum detection.

Radioelectronic Systems Conference 2019, edited by Piotr Kaniewski, Jan Matuszewski, Proc. of SPIE Vol. 11442, $1144212 \cdot$ (c) 2020 SPIE · CCC code: 0277-786X/20/\$21 · doi: 10.1117/12.2565720 


\subsection{Selected aspects of quantum remote sensing}

In classical remote-sensing methods, most frequently used systems are based on measurements of electromagnetic radiation properties spontaneously emitted or deflected by monitored objects. Lighting from sources emitting monochromatic beams or having specific spectral characteristics is used for this purpose. Remote sensing surveillance is carried out on all technically feasible electromagnetic wavelengths and polarizations. The first sources used were natural, but with the development of technology, artificial sources of electromagnetic radiation were introduced. An indispensable element of every remote sensing system is an electromagnetic radiation detector. The development of the existing remote sensing methods was based on the principles of traditional physics, but with the discovery of physical bases of thermal radiation and light and understanding of the laws describing the process of generation and emission of electromagnetic radiation, it was possible to introduce quantum techniques.

The views on the nature of heat and light were formed while observing the phenomena occurring during the generation and transmission of energy, and studying the incandescence of bodies during their heating and cooling. Problematic interpretations of the results of the experiments divided scientists into two opposite camps: one supported the wave nature of light and the other backed the corpuscular theory. As a result of centuries of discussions, but also of works by de Broglie, Bohr, Maxwell, Schrödinger, Heisenberg, Einstein and other scientists, the perception and understanding of light as being of dual nature classified as a corpuscular-wave dualism - both as an electromagnetic wave and as a stream of particles called photons - has been formulated.

The concept of quantum and quantum properties of heat and light introduced by Planck, as well as the equations describing intensity and spectral distribution of thermal radiation of a black body became strong foundations for the controversial quantum theory. Einstein's philosophical considerations on physical phenomena and mental processes laying foundation for the act of observation led to the famous thought experiment of Einstein-Rosen-Podolsky (EPR), the interpretation of which was aimed at providing arguments to demonstrate how incomplete or even erroneous quantum theory of Planck has been [2].

Surprisingly, however, the EPR experiment not only failed to refute quantum theory, but also detected new, inexplicable effects. The logic of the experiment was based on observation of particles emitted in pairs (in singlet state) or photon pairs. After the separation of particles in relation to the direction of their movement, the measurements of the quantum state (spin) of some pairs of particles showed a correlation. Each measurement could give two results - the same or opposite spin - but until seeing the measurement result, the state of each component (of the two particles forming the object) was equally probable and remained unspecified. The particles of such an object were called to be in the state of quantum entanglement. The discovery proved that becoming conscious of the state of one of the elements of the object, which is in a quantum entanglement relation with another element of the object, determines and requires the state of the other element, which would have been observed at the moment of making a corresponding measurement on this element, without making any direct observations.

The aforementioned circumstance seems completely absurd on the grounds of traditional mechanics, however, the unreliability of intuitive predictions and interpretation of the results of observations based on traditional mechanics was demonstrated by a mental experiment by J. S. Bell's, called the Bell paradox [2]. Verification of Bell's hypothesis was tested in a physical experiment of Aspect (1982), the diagram of which is shown in Figure 1. 


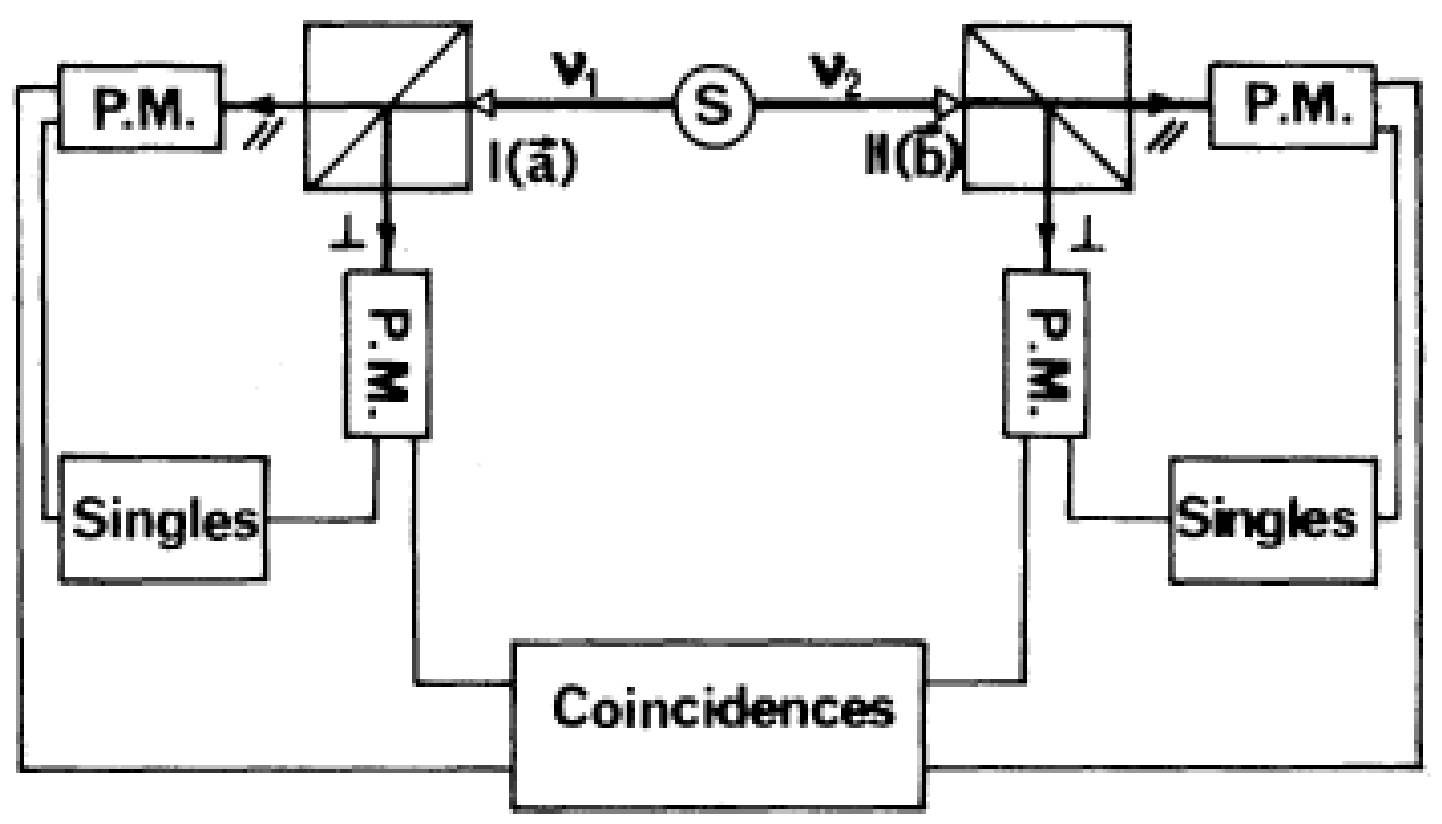

Figure 1. Schematic diagram of Aspect's experiment testing Bell's inequalities [4]

In this system, the source 'S' (excited calcium atom) simultaneously emits two identical optical photons, $v 1$ and $v 2$, but in opposite directions, while two identical polarizers select the emitted photons according to the established polarizations in such a way that all photons of horizontal orientation as well as all photons of vertical orientation are counted separately for each direction and orientation with appropriate photomultipliers (P.M.). The received signals were sent to the coincidence recorder at the same time. Such a system tests different physical situations resulting from the activities of observing photon production. A corresponding situation will occur in the system shown in Fig. 2. when as a result of a photon and optically nonlinear material interaction, the photon becomes a source simultaneously emitting two identical photons, but with a total energy equal to the energy of one photon (HW - halfwave).

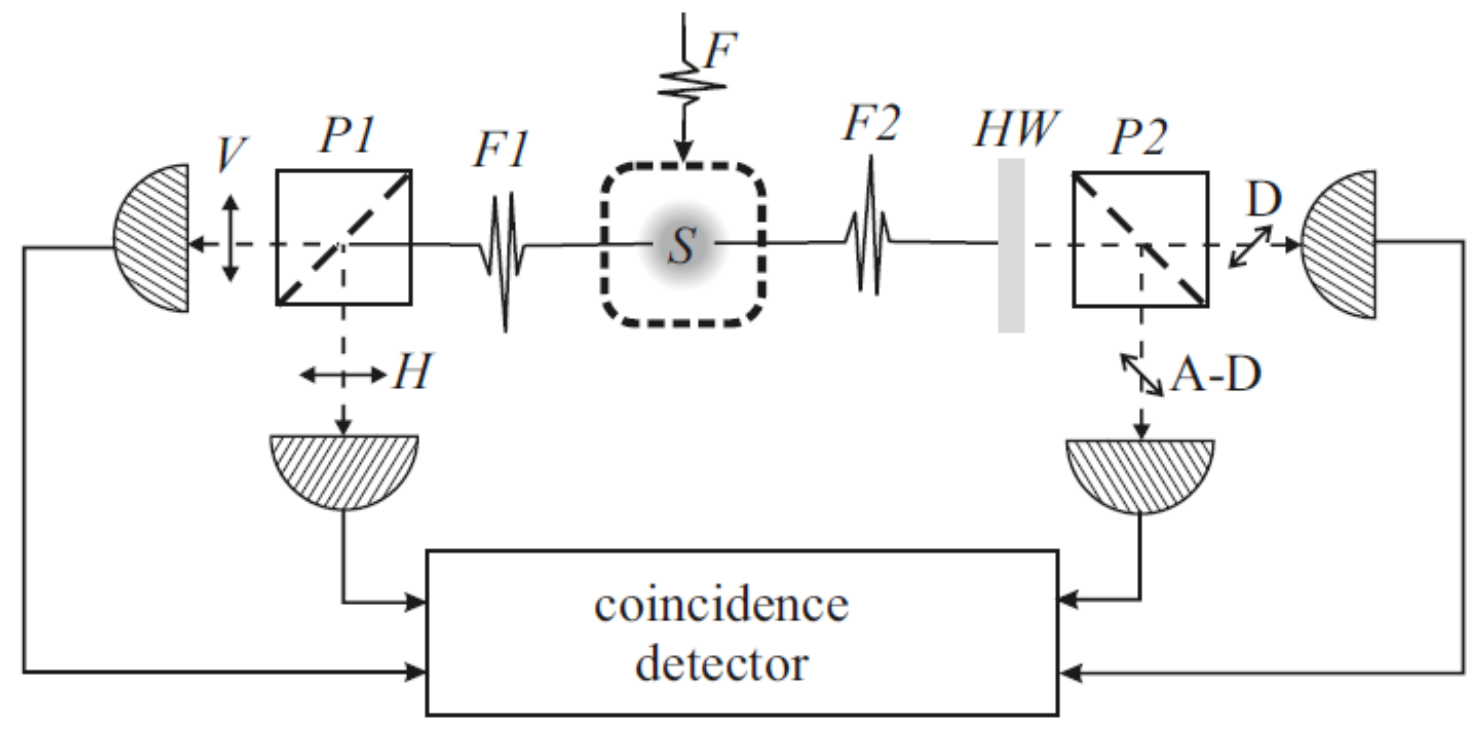

Figure 2. Schematic diagram of the modified Aspect's experiment 
The analysis of possible states that the receiver identifies is connected with the problem of individuality and distinguishability of identical objects (e.g. photons). This problem is the continuation of one of the philosophical inquiries made in the 12th century by Duns Scotus, which was expressed in the following sentence: "There are no two indistinguishable individuals (...). If two things are indistinguishable, then the same thing is called by two names".

Since photons are not distinguishable entities in traditional terms and are not subject to Maxwell-Boltzmann's statistics, their detection and measurement should be considered in many aspects - the category of the foundation of the act of observation as well as the category of measurement and unambiguous interpretation of the information obtained. Given the fact that this background gives rise to logical conflicts of ambiguous assessment of information, the result is a paradox. Permanent research carried out in this area resulted in the discovery of further quantum phenomena - quantum entanglement and quantum teleportation, which became the basis for cryptography, quantum calculations and quantum remote sensing.

The state of entanglement results from the consequences of quantum mechanics, which permits the existence of a quantum system in several states simultaneously, corresponding to different values of physical observability such as the position, momentum or spin. Changes in the properties of entangled elements, e.g. photons, are correlated, and in a compound system (e.g. of two, three or more elements) they are bound together inseparably in relation to each other, even if the individual elements of such an object are spatially separated.

From the traditional point of view, entangled states seem to be a paradoxical necessity of simultaneous existence of a specific being or system at the same time and in many places. Currently quantum mechanics treats physical bodies as objects that can be described as the so-called wave function, while the state of quantum entanglement explains the situation in which there is a correlation of these functions (i.e. the occurrence of quantum correlation between physical objects). The effect of quantum entanglement between completely separate objects, e.g. single photons, atoms or molecules, is the manifestation of phenomena that are never encountered in traditional systems - e.g. it is assumed that the impact on one of the components of an object composed of elements connected with quantum entanglement is transferred without any delay over any possible distance to the other component. The effect of non-locality of phenomena, i.e. the immediate impact of phenomena taking place in one location in space on a phenomena in another location space, is the non-locality of information and non-locality of interpretation of information. The quantum state in which one of the elements of the entangled system is found before the measurement determines the quantum state of the other element (and vice versa), regardless of how distant these elements are from each other. Accordingly, the act of measuring the state of one of the elements - e.g. the observation of polarization - determines the state of the other element.

The recognition of the existence of quantum entanglement opened the possibility of remote sensing surveillance by means of photons entangled with photons illuminating the object [5]. The use of entangled photons in remote sensing unexpectedly creates surprising possibilities of detecting and imaging objects while maintaining the observation entirely undetected [6].

\section{SOURCES OF ENTANGLED PHOTONS}

The beams containing pairs of entangled photons were initially produced by a type II parametric fluorescence process, also known as spontaneous parametric downward conversion or parametric scattering. The process of type II parametric fluorescence is carried out in accordance with the principles of energy and momentum conservation, e.g. when nonlinear optical materials, e.g. $\mathrm{BBO}$ crystals i.e. barium $\beta$-borate $(\beta(\mathrm{Ba}(\mathrm{BO} 2) 2)$, are illuminated with an intensive laser beam. Entangled photons are produced using the energy of one photon, called the pumping photon, which as a result of interaction with the BBO crystal, transforms into two photons with lower energy, i.e. signal photon and no-load photon, the total energy of which is equal to the energy of the pumping photon - in other words, the conversion process presented in the name is carried out downwards.

Unlike type 0 parametric fluorescence, where the photons produced maintain the same polarisation as the pumping photon, and type I parametric fluorescence, where the photons produced have polarisation perpendicular to the polarisation plane of the pumping photon, the polarisation planes of photons produced in the type II parametric fluorescence are always perpendicular in relation to each other (fig. 2). In the process of producing entangled photons based on type II parametric fluorescence, most of the photons hitting the crystal pass through it without any change. 
Only a few photons "pumped" into the crystal spontaneously and instantly transform into a pair of entangled photons. As the processes of the production of entangled photons are very probabilistic in nature, the sources based on the above principle are characterized by very low efficiency.

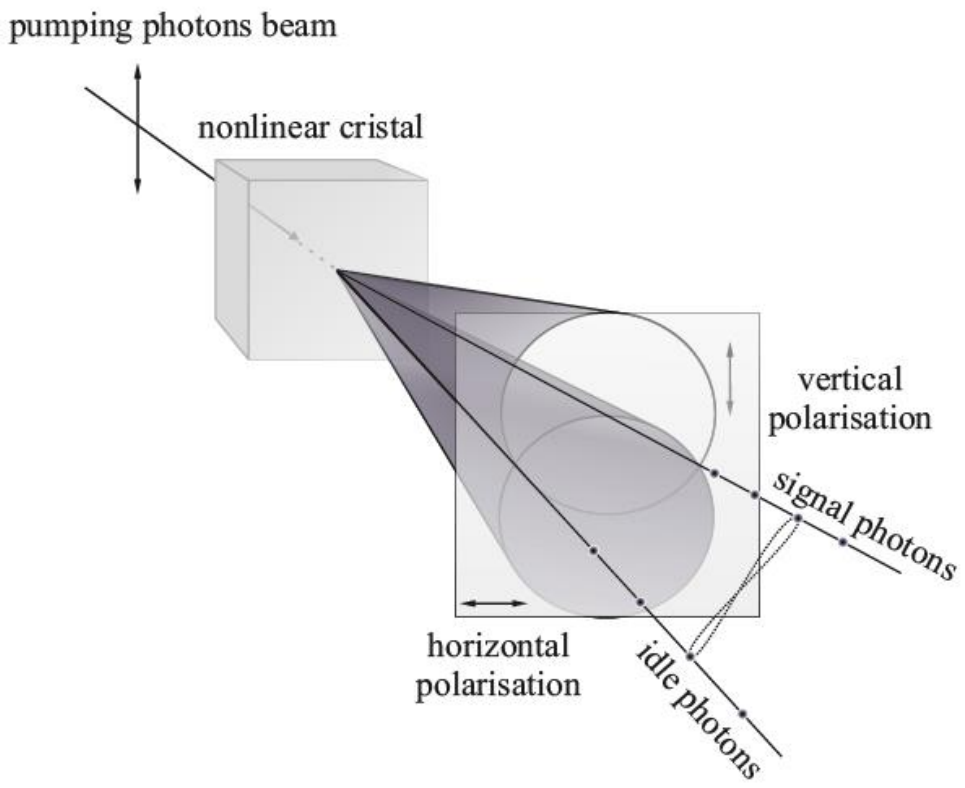

Fig. 3. Schematic diagram of geometric relationships of beams during the production of entangled photons

A source of entangled photons which is much more efficient are electroluminescent diodes and semiconductor quantum dots, however, extraction of entangled photons from such sources faces a serious technical problem [7, 8]. The efficiency (at the level of 65\%) of extracting entangled photons from the beam emitted from quantum dots was demonstrated in the system with a broadband optical emitter [9]. There are known reports on applications of ultra-cold atoms (Bose-Einstein condensate) as a source of entangled photons in remote sensing systems [10,11]. Sources as such require cryostat and cryogenic technology with liquid helium.

\section{Applications of entangled photons}

The entangled states can be used for both encryption and distribution key generation, which has contributed to the development of quantum cryptography. Based on the laws of quantum mechanics, the decryption key can be transmitted and read completely securely, even in the presence of tapping devices. Any attempt to intercept quantum encrypted information in an unauthorised manner is fundamentally ineffective.

Quantum key techniques led to the concept of using quantum entangled objects in remote sensing. Two visions of quantum radar were created: the first - providing solution to the problem of radar interference, is based on the concept of using the mechanism of quantum key distribution, while the second - significantly improving the resolution of the radar, is based on the technique of detection by means of the state of entanglement. Achieving these images in the radar permits to reach the super-resolution quantum at the Heisenberg limit.

In conventional remote sensing, imaging is performed using "normal" photons (from the environment or from an artificial source, e.g. an illuminator), the photons deflected from the object they illuminate are recorded e.g. by a camera. In quantum remote sensing, objects are not illuminated by "ordinary" photons, but by 
photons that are parts of a pair of quantum entangled photons. For this purpose, the produced entangled photons are divided into two separate beams, one of which is directed towards the observed object and the other one is kept in the receiver. As a result of the preserved quantum consistency there is no interference observed while illuminating the object with "ordinary" photons. The interaction of entangled photons and the body illuminated by them remains entangled until they have been consciously registered.

Although the efficiency of the entangled photons production process is usually at the level of a few pairs of entangled photons per million other "ordinary" photons, the illumination with the application of single photons is still effective in sensing the object illuminated by them. The illumination by the quantum entangled photon beam is fundamentally different from that by the 'ordinary' photons. The interaction of entangled photons and the body illuminated by them maintains the entangled state. As a result of the preserved quantum consistency there is no interference observed while illuminating the object with "ordinary" photons.

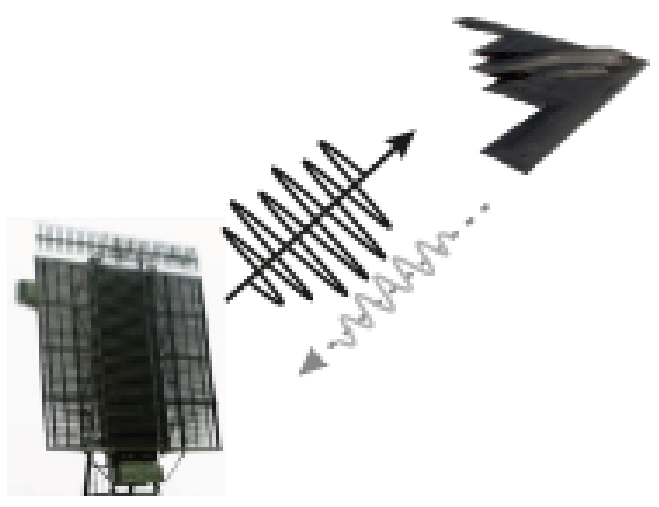

Figure 4. The principle of the traditional radar operation

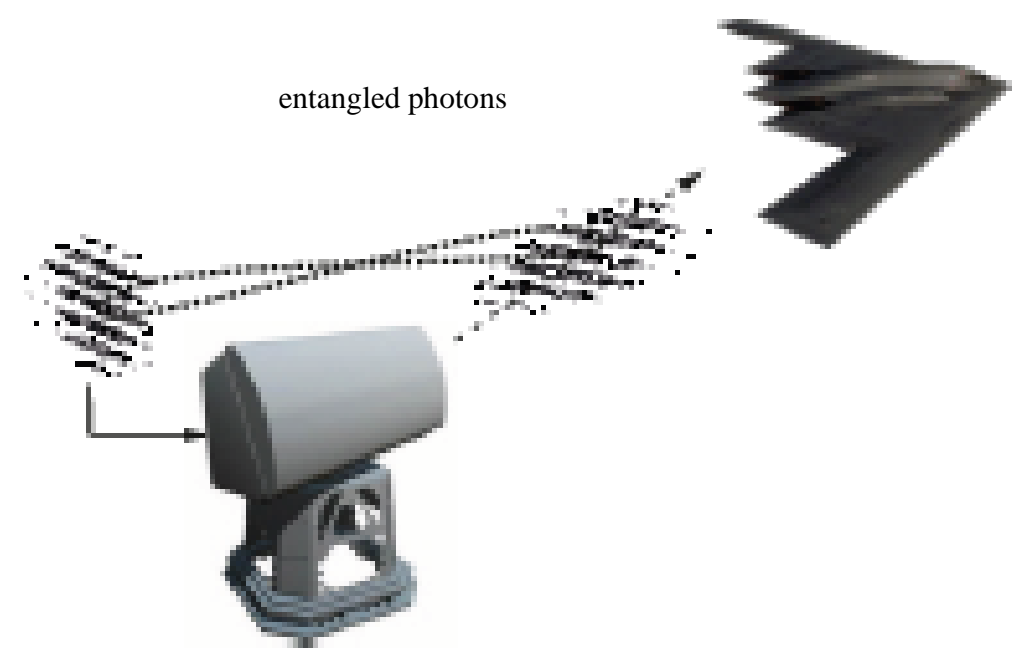

Figure 5. The principle of a quantum radar operating with entangled photons. [14] 
Object imaging with the application of the entangled photons is based on combining information collected by two separate detectors, e.g. a multi-pixel matrix that "does not see" the object and a single-pixel sensor that "sees" the object. By combining data from both detectors, a high signal-to-noise ratio is achieved. The earliest description and conceptual scheme of a device designed for the quantum imaging of objects (the so-called quantum radar) is presented in the patent description [15] as well as in the most recent articles [16]. The detailed operating rules are disclosed in patent No CN201710036608.3A. As described above, the quantum radar consists of a transmitting and receiving station mounted on a rotating bracket, figure 6 .

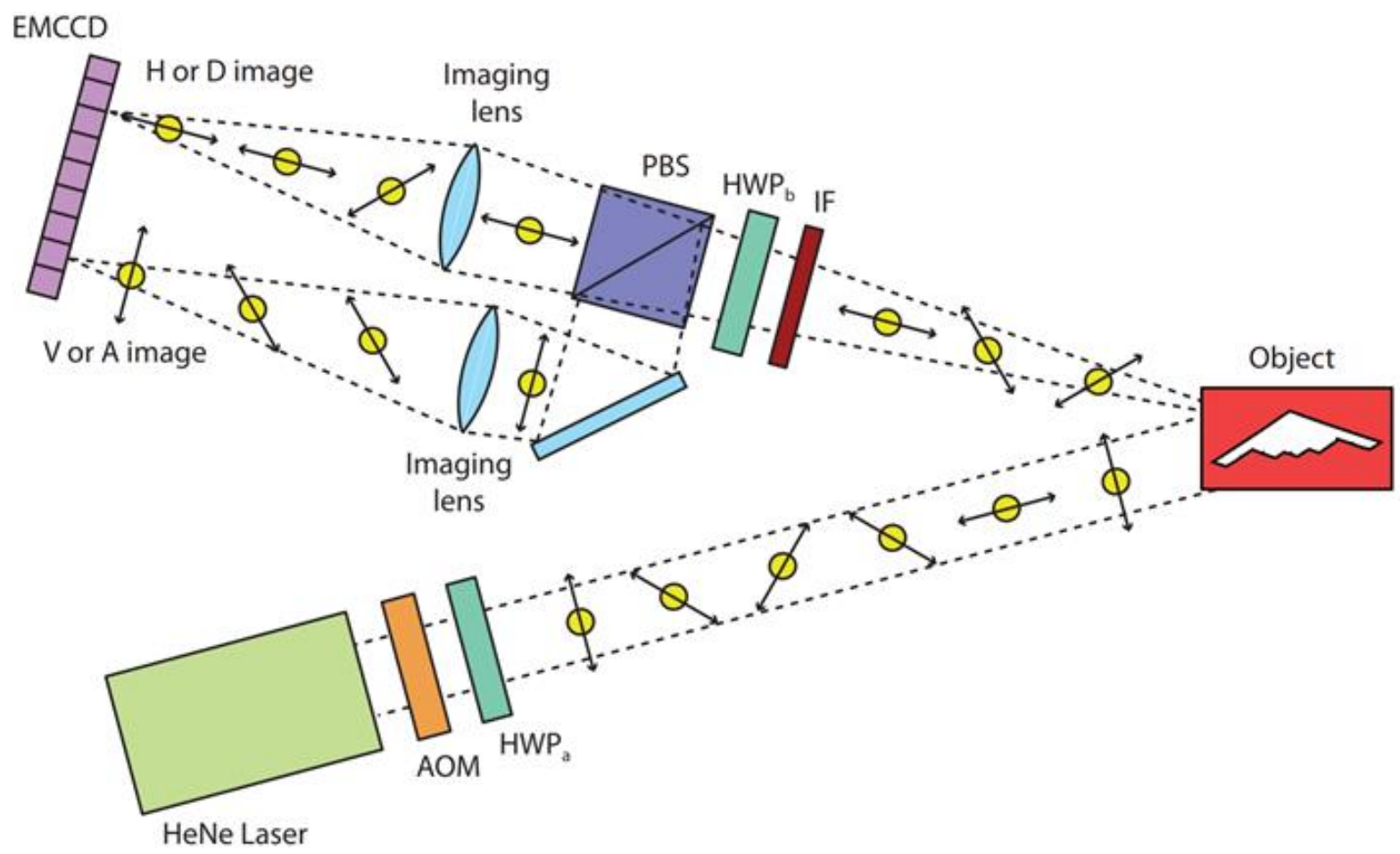

Fig. 6. Principle of object imaging used in a quantum radar [16]

The device uses a helium-neon laser emitting a laser beam, with which an acoustic-optical modulator (AOM) forms pulses containing a certain number of photons. These impulses are then passed through a rotating half-wave plate (HWPa), and as a result, the orientation of photons is randomly assigned to one of four polarization cases: horizontal ( $\leftrightarrow$

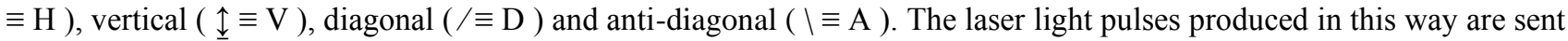
to the monitored area. On the part of the receiving station, if there is an object in the observed space, some of the photons are absorbed or deflected by the object. As a result, both background and deflected photons reach the receiver. The interference filter (IF) is used to remove ambient photons (noise). Then, in order to measure the polarization of the received light pulses, the photons are directed to a half-wave plate (HWPb) and then to a polarization beam splitter (PBS).

When the light of the received light pulses is parallel to the direction of vertical polarization $(\uparrow)$, the polarizing beam splitter (PBS) is also in a vertical polarization $(\uparrow)$ and when the polarization is horizontal $(\leftrightarrow)$, the polarization beam splitter (PBS) is also in a horizontal polarization $(\leftrightarrow)$. When the half-wave plate and the angle between the orientation of vertical polarization is $22.5^{\circ}$, the measuring light pulse has either a diagonal ( / ) or anti-diagonal ( $\backslash$ ) polarization. The lenses on both sides of the polarization beam splitter (PBS) send the split measurement light pulses in relation to the 
polarization orientations towards the receiving transducers - however, each orientation of polarization is directed to the appropriate CCD transducer (EMCCD) with electron multiplication. The description of the device claims that it is able to reliably detect objects from virtually any distance using only one pair of entangled photons and the phenomenon of polarization. Although the efficiency of the entangled photons production process is usually at the level of a few pairs of entangled photons per million other "ordinary" photons, their extremely low numbers are still effective in sensing the object illuminated by them. The examples of aircraft and bird images are shown in Figure 7.

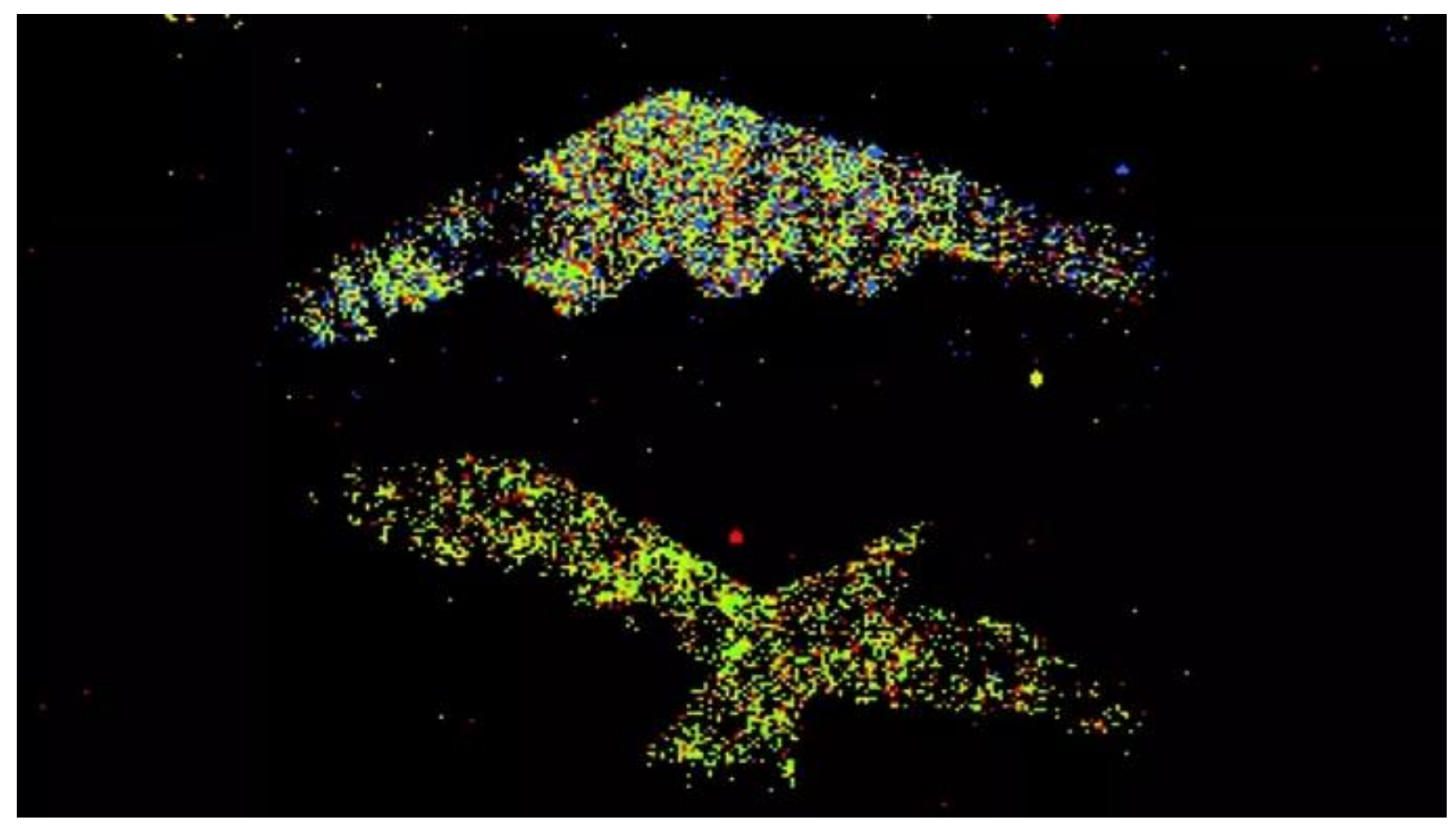

Fig. 7. The examples of recorded images of an aircraft and a bird [16]

The difficulty or even impossibility of detecting an object with a built-in stealth technology by a traditional radar device results both from the fact that such an object absorbs a part of the radar's radiation falling on its surface and from the fact that it deflects and directs the radar beam in directions other than the direction from which the radar beam came from. Quantum radar overcomes this difficulty because it does not receive echoes of signals deflected from the target surface. 
(a) Secure Image

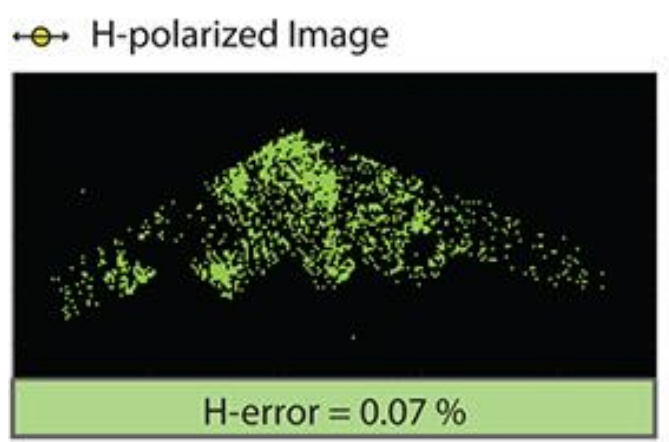

$\varnothing$ D-polarized Image

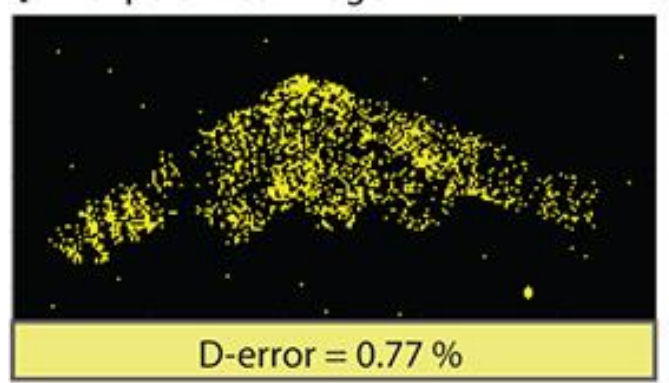

(b) Compromised Image

$\rightarrow$ H-polarized Image

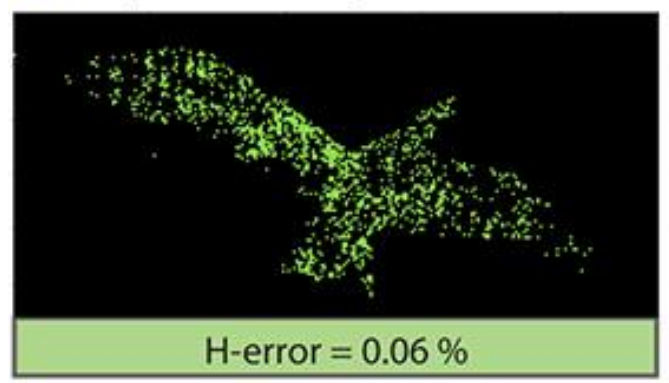

\section{$\varnothing$ D-polarized Image}

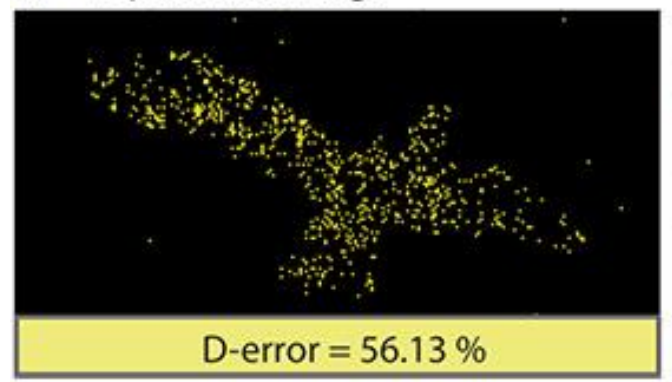

$\oint$ V-polarized Image
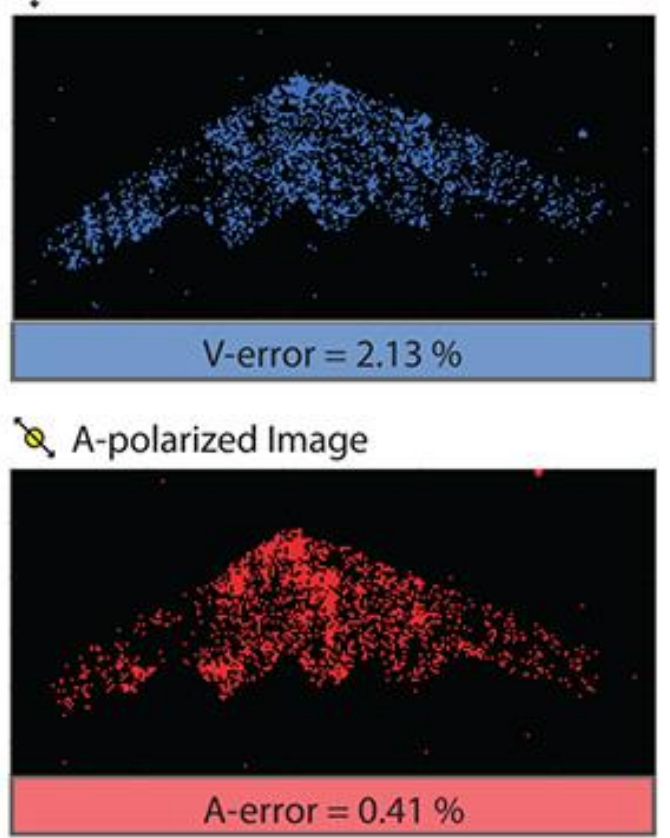

$\oint$ V-polarized Image

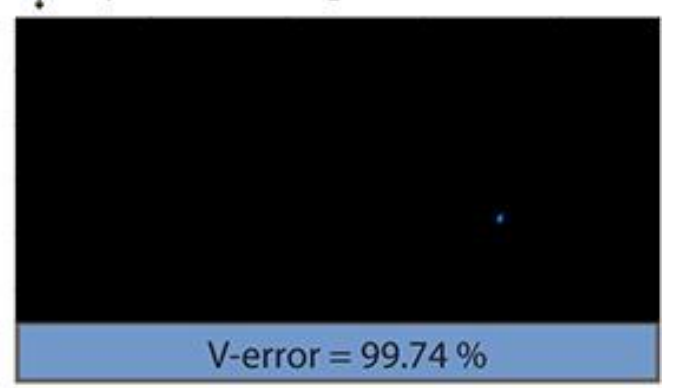

Q. A-polarized Image

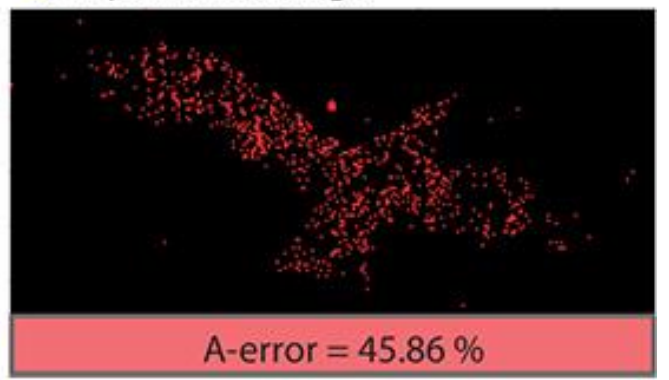

Fig. 8. The examples of recorded images of an aircraft and a bird for different polarization orientations [16] 
Related to quantum remote sensing is a technique called "ghost imaging" that allows you to get an image of objects that the camera itself cannot see. This technique uses two sensors, one of which observes the light source and the other observes the object. Both sensors point in different directions. One of the sensors (high resolution camera placed away from the object) is directed towards the light source, while the other sensor is directed towards the object (one-pixel sensor, like a photodiode).
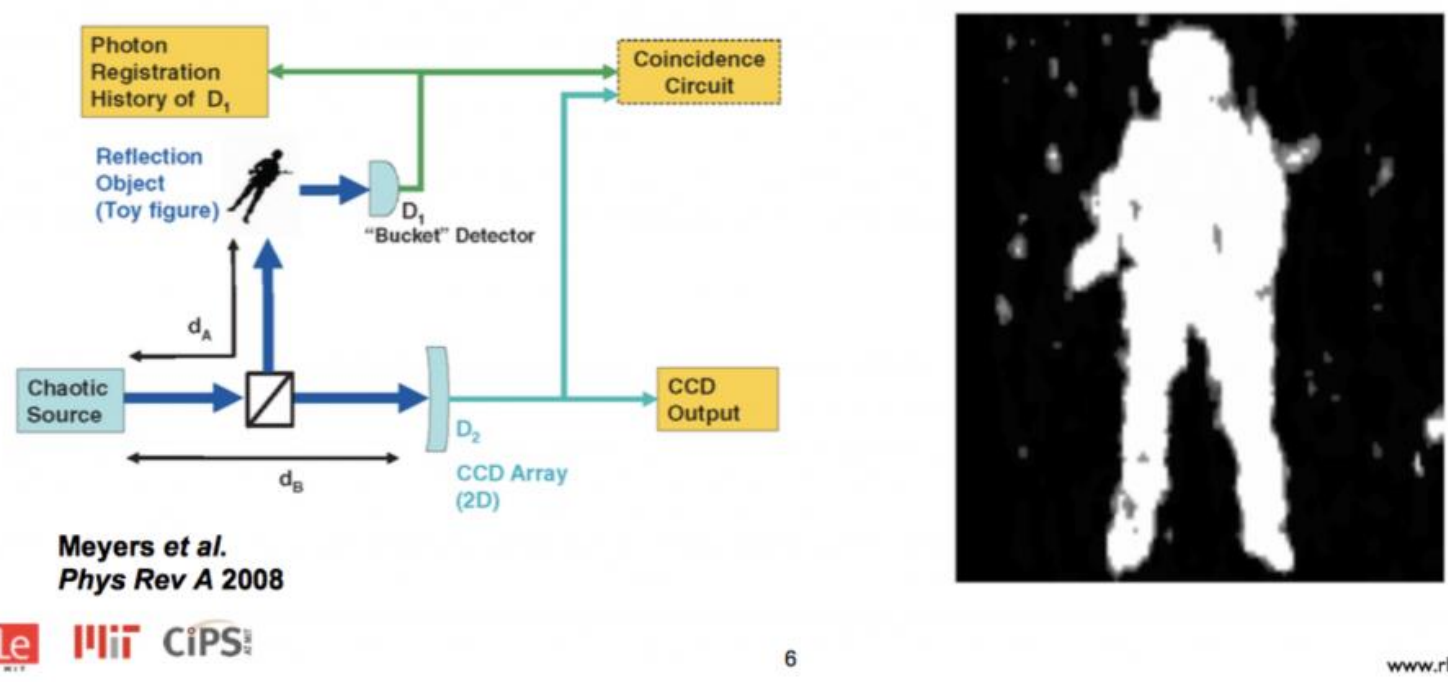

Figure 9. Setup of "ghost image" registration system and example of recorded image [18 ]

The photo shows the first "ghost image" of an opaque object (a toy soldier), which was registered on the University of Maryland, Baltimore, US Army Research Laboratory.

\section{CONCLUSIONS}

First theoretical considerations and mind experiments on the understanding of the impact of the act of observation and measurement on the measurement result led to the discovery of a phenomenon called the quantum entanglement. Further experimental work led to the corroboration of the hypothesis that there is an inseparable link between observations and the result of measurement. The first practical application of the quantum entanglement phenomenon was the transmission of information in such a way as to ensure that the transmission channel is completely hermetic. The effects of quantum entanglement in remote sensing seem to be even more spectacular. Test results from the first experimental quantum detectors have shown that observations made with their use permit reliable detection of previously undetectable objects, while retaining their own complete undetectability and concealment. So far, a small number of patent applications in this area (about 40) show it is only the beginning of the race. The USA and China are in the leading positions.

Due to the use of wavelengths (photon energies) in the current structures of quantum radars, their range is relatively small (optical). From the point of view of radiolocation, we need a radar system capable of detecting small objects, simultaneously combining the capabilities of existing systems that result from the long wave propagation properties. Considering that it is possible to create the state of entanglement of many photons (the so-called multi-photon) which corresponds to the wavelength of the sum of wavelengths of entangled components of photons, it will probably be possible to produce such a system. The superiority of a quantum radar over a traditional radar is manifested in a lower risk of signal detection and extremely high resolution. 
This technology will indisputably raise the rate of object detection and drastically reduce the opponent's defensive capabilities. In such circumstances, the necessity to conduct own extensive and omni-directional research in this area seems an obvious step to be taken.

\section{REFERENCES}

[1] Bi Siwen, Quantum remote sensing theory and practice, Proceedings of the SPIE, Volume 10765, id. 1076505 27 pp. (2018).

[2] A. Einstein, B. Podolsky N. Rosen, Phys. Rev. 47, 777 (1935).

[3] J. S. Bell, „On the Einstein Podolsky Rosen Paradox”, Physics 1 (1964) 195-200

[4] A. Aspect, P. Grangier, G. Roger, „Experimental Realization of Einstein-Podolsky-Rosen-Bohm Gedankenexperiment: A New Violation of Bell's Inequalities”, Phys. Rev. Let., V49, 2, (1982)

[5] Einstein i in. „Can quantum-mechanical description of physical reality be considered complete?”

[6] J. L. O'Brien, A. Furusawa i J. Vuckovic, „Photonic Quantum Technologies”, Nature Photon. 3 , 687 (2009)

[7] Dousse, A. i in., „Ultrabright source of entangled photon pairs”. Nature 466 , 217-220 (2010).

[8] C. Emary et al., „Entangled Microwaves from Quantum Dots”, arXiv:cond-mat/0502550 v1, pp. 1-4, Feb. 23, 2005.

[9] Yan Chen, Michael Zopf, Robert Keil, Fei Ding, Oliver G. Schmidt, „Wavelength-tunable entangled photons from silicon-integrated III-V quantum dots",

[10] Nature Communications, 9 , 2994 ( 2018 ).

[11] A. Sørensena, L.-M. Duan, JI Cirac i P. Zoll, „Many-particle entanglement with Bose-Einstein condensates” Nature 409, 63 (2001).

[12] K. Helmerson, Li You, "Creating Massive Entanglement of Bose-Einstein Condensed Atoms”, Phys. Rev. Let. 87, 17, Oct. 22, 2001.

[13] Fu Wei Zhu Wei, (2017) patent nr CN106680828A "Quantum radar based on quantum correlatin and processing method thereof".

[14] Ioannou. (2018) "Review on quantum radar" Conference Paper, Jan. 2018, 6th International Conference on Experiments/Proces.

[15] Yan Chen, Hongyu Yang, Uui Zhao, „Quantum radar technology and its developments” ICC 2013, DOI: 10.1109/icccas.2013.6765317.

[16]E.H. Allen, M. Karageorgis, (2019) patent nr US7375802B2 (2019) "Radar system and methods using entangled quantum particles".

[17] Mehul Malik, Omar S. Magana-Loaiza, Robert W. Boyd, "Quantum-secured imaging”, Applied Physics Letters 101, 241103 (2012).

[18] Miller G. , "By uncertain light: the use of quantum mechanics -. Ghost imaging and ability to capture with limited light", Teledyne imagining < https://possibility.teledyneimaging.com/boo-have-you-seen-ghostimaging/ (2017). 